\title{
Is the Non-Alcoholic Fatty Liver Disease Part of Metabolic Syndrome?
}

\author{
Sur Genel ${ }^{1,2 *}$, Coroian Aurelia ${ }^{4}$, Valer Donca ${ }^{1,3}$ and Floca Emanuela ${ }^{1}$
}

${ }^{1}$ University of Medicine and Pharmacy, Iuliu Hatieganu, Cluj-Napoca, Romania

${ }^{2}$ Emergency Clinical Hospital for Children, Cluj-Napoca, Romania

${ }^{3}$ Municipal Hospital, Cluj Napoca, Romania

${ }^{4}$ Faculty of Animal Science and Biotechnology, University of Agricultural Sciences and Veterinary Medicine Cluj-Napoca, Romania

\begin{abstract}
Non-alcoholic fatty liver disease (NAFLD) is caused by fatty infiltration of the liver in the absence of alcohol consumption. The incidence of NAFLD in general population is $30-40 \%$. NAFLD is considered by some authors to be the liver manifestation of the metabolic syndrome and by other authors is considered to be a separate entity. The pathogenesis of NAFLD and metabolic syndrome seems to have common pathophysiological mechanisms, with focus on insulin resistance as a key factor. Insulin resistance seems to explain the accumulation of triglycerides in the liver, being the main pathogenetic link involved in the onset and progression of NAFLD. The natural evolution is not well known, and there is some controversy about the progression from NAFL to NASH and fibrosis. Current data on epidemiology, pathophysiology and diagnostic supports the association of non-alcoholic fatty liver disease as a possible component in the cluster of metabolic syndrome. Clinical, experimental and epidemiological studies argue that NAFLD may be the hepatic manifestation of metabolic syndrome.
\end{abstract}

Keywords: NAFLD; Metabolic syndrome; fatty liver

\section{Introduction}

Non-alcoholic fatty liver disease (NAFLD) is the most prevalent liver disease. Nonalcoholic fatty liver disease (NAFLD) refers to a spectrum of liver damage ranging from simple steatosis to nonalcoholic steatohepatitis (NASH), advanced fibrosis and cirrhosis [1].

There are other factors and conditions that can lead to fatty liver, called non NASH fatty liver (NNFL), such as lipodystrophy, Wilson's disease, primary mitochondrial disease, bariatric surgery, parenteral nutrition, medication (amiodarone, methotrexate, tamoxifen), toxins. The incidence of NAFLD varies on the geographic area and the diagnostic method. In Europe, the NAFLD incidence in the general population is $20-30 \%$ and in the USA is $27-38 \%$. In Europe for diagnostic is used ultrasonography and in USA is used histology $[2,3]$.

NAFLD is associated with components of the Metabolic Syndrome (MS) such as abdominal obesity, insulin resistance, dyslipidemia, glucose intolerance or type 2 diabetes mellitus (T2DM). The insulin resistance was identified as a central point in pathogenetic mechanism of NAFLD, so it can be considered the liver manifestation of the MS. Insulin resistance in NAFLD is characterized by reduced whole-body, hepatic, and adipose tissue insulin sensitivity. Insulin resistance is often associated with chronic low-grade inflammation, and numerous mediators released from immune cells and adipocytes may contribute liver damage and liver disease progression. In the MS the major associations of NAFLD are with obesity and with T2DM. The patients with these disorders have a $70-90 \%$ prevalence of NAFLD. Patients with NAFLD and T2DM have an additional risk of developing NASH, advanced fibrosis and cirrhosis and hepatocellular carcinoma, chronic kidney disease and retinopathy $[1,4,5]$.

MS is a set of metabolic and cardiovascular risk. The MS has been defined in 2009 by presence of a least three of the following five criteria: abdominal obesity (waist circumference increased for the Europeans $\geq 94 \mathrm{~cm}$ in men and $\geq 80 \mathrm{~cm}$ in women; for the Americans $\geq 102$ $\mathrm{cm}$ in men and $88 \mathrm{~cm}$ in women), elevated triglycerides $>150 \mathrm{mg} / \mathrm{dl}$ or treatment for hypertriglyceridemia, low HDL cholesterol $<40 \mathrm{mg} /$ dl or treatment for low HDL cholesterol, hypertension $>135 / 85 \mathrm{~mm}$
$\mathrm{Hg}$ or treated hypertension, high fasting plasma glucose $>100 \mathrm{mg} / \mathrm{dl}$ or treatment for hyperglycemia [6-8].

Over $90 \%$ of patients with NAFLD have at least one component of the MS and the complete diagnosis of MS is present at 55-65\% of the patients with liver disease.

\section{Definition and natural history}

In 1980 Ludwig et al. coined the term Non-Alcoholic SteatoHepatitis (NASH) to describe the morphological pattern of liver injury. The natural evolution is not fully known and there are some controversies about the progression from NAFL to NASH and fibrosis. Some studies demonstrated that in the presence of simple steatosis (NAFL) the mortality is not different from what is recorded in the general population. If steatosis with inflammation is present (NASH) there is an increased mortality. The risk of progression in HCC in many studies showed that NAFLD is the most common etiology of HCC $[1,9]$.

NAFLD is the third cause of liver transplantation in US after infection with HCV and the Alcoholic Liver Disease (ALD). Introduction of new therapies for HCV infection make NAFLD the main etiology for liver transplantation [10].

\section{Conditions associated with fatty liver disease}

Many different agents and conditions have been associated with fatty liver disease: $[4,11,12]$

${ }^{*}$ Corresponding author: Dr. Genel Sur, Emergency Clinical Hospital for Children, Cluj-Napoca, Romania, E-mail: surgenel@yahoo.com

Received January 30, 2015; Accepted March 19, 2015; Published March 21 2015

Citation: Genel S, Aurelia C, Donca V, Emanuela F (2015) Is the Non-Alcoholic Fatty Liver Disease Part of Metabolic Syndrome? J Diabetes Metab 6: 526 doi:10.4172/2155-6156.1000526

Copyright: $\odot 2015$ Genel S, et al. This is an open-access article distributed unde the terms of the Creative Commons Attribution License, which permits unrestricted use, distribution, and reproduction in any medium, provided the original author and source are credited. 
1. Acquired insulin resistance: obesity, diabetes mellitus, hyperlipidemia, hypothalamic - pituitary dysfunction.

2. Genetic - inborn errors of metabolism: abetalipoproteinemia, Weber-Christian disease, galactosemia, limb lipodystrophy, type 1 glicogen storage disease, Wilson disease, tyrosinemia, systemic carnitine deficiency, Refsum syndrome.

3. Nutritional and intestinal disorders:

- Surgical: jejunal bypass, gastroplasty for morbid obesity, biliopancreatic diversion, extensive small bowel rejection;

- Inflammatory bowel disease;

- Protein caloric malnutrition

- Cachexia;

- Rapid weight loss, total parenteral nutrition, jejunal diverticulosis

4. Drugs and toxins: methotrexate, amiodarone, glucocorticoids, Tomoxifen, calcium channel blockers, phosphorus organic solvents, petrochemicals.

The exact pathogenesis of NAFLD remains poorly understood. Insulin resistance plays a central role in the net retention of lipids, particularly triglycerides by the hepatocyte. Insuline resistance has consequences in the metabolism of fatty acids and carbohydrates, resulting in fatty liver, changes that, in turn, lead to the aggravation of insuline resistance [13-15]

The second cause is generally atribuited to oxidative stress wich causes peroxidation of lipid in the hepatocyte membrane, cytokine production and Fas ligand induction, beeing responsible for the progression from steatosis to NASH to cirrosis. The oxidative stress leads to mitochondrial dysfunction and to an inadequate response of the endoplasmic reticulum, and mitochondrial dysfunction and mitochondrial destruction are associated with insulin resistance.

\section{Diagnosis}

\section{Signs}

There are no pathognomonic signs of NAFLD. Obesity is present in $30-100 \%$ of patients during physical examination. Hepatomegaly occurring in $45 \%$ of patients and signs of portal hypertension (splenomegaly, spider nevi and palmar erithema) are the most common symptoms. Most patients with NAFLD (48-100\%) are asymptomatic $[1,11]$.

Vague right upper quadrant abdominal pain, fatigue and malaise are the most common of these nondescript symptoms. Pruritus, anorexia, nausea, jaundice, abdominal distension (ascites), gastrointestinal bleeding, confusion (encephalopathy) are all indicative of advanced liver disease occurring late in the course. The liver disease is often discovered incidentally when a hepatic panel reveals an elevated ALT level [1].

\section{Laboratory}

Moderate elevation of serum aminotransferase (ALT and AST) is the most common. It is important to exclude secondary causes of hepatic fat.

Liver biopsy is the only accurate method for the diagnosis of NASH and to determinate the severity of liver damage and long term prognosis [7].
There are two lesions associated with NAFLD: predominantly macro-vesicular steatosis alone or associated with varying amounts of cytological ballooning and spotty necrosis coffered mixed, neutrophilic lymphocytic inflammation glycogen nuclei.

The severity of steatosis can be graded on the basis of the extent of involved parenchyma. The most useful classification is the Bunt's classification that unifies the steatosis and necroinflamation of fibrosis into the stage. According to the Bunt's classification there are 3 grades of steatosis and 4 grades for fibrosis.

\section{Treatment}

\section{Weight management}

Many studies show that moderate sustained and gradual weight loss may lead to an improvement in liver biochemistries and histology.

\section{Insulin resistance}

Pharmacologic agent controls insulin resistance. Thiazolidinediones favour the stimulation of peroxisome proliferator-activated receptor- $\gamma$. Metformin (a biguanide) is another antidiabetic medication for hepatic insulin sensitivity.

\section{Lipid lowering agents}

Hypertrigliceridemia is often associated with NAFLD. Clofibrat ( $2 \mathrm{~g} /$ day) had no beneficial effect in liver tests or hepatic histology. Hepatocite protection is often a therapeutic agent such as ursodeoxycholic acid (UDCA), anti-oxidants, betaine and Vitamin E.

Patients with NAFLD who develop end stage liver disease should be evaluated for liver transplantation.

\section{Metabolic syndrome and NAFLD}

Recent studies have shown that NAFLD being manifested by simply fat liver to NASH may represent another feature of the metabolic syndrome. Insulin resistance and hyperinsulinemia play a central role both in NAFLD and metabolic syndrome. Through clinical manifestations and laboratory data such as normoglycemia and a moderate increase in body weight, NAFLD has similar characteristics with diabetes and obesity. Studies have pointed out that almost all patients (90\%) with NAFLD have at least one risk factor of MS and that about one-third of them have full features of MS. Obesity is present in $30-100 \%$ of patients with NAFLD and type-2 diabetes in $10-75 \%$. Hyperlipidemia, hypertriglyceridemia and low HDL cholesterol levels are strongly associated with NAFLD. Recent studies have shown that NAFLD increases the risk of cardiovascular disease. In a prospective observational study Hamagush found that MS is a risk factor for NAFLD [1].

\section{Conclusion}

NAFLD is an important chronic liver disease. Insulin resistance and oxidative stress play critical role in pathogenesis and this mechanism is met in MS. NAFLD is strongly associated with obesity and T2DM as part of MS. Treatment aimed at reducing weight, triglycerides, and insulin resistance. NAFLD can be considered part of MS or an associated factor. Studies that clarify the pathogenic mechanism are needed leading to the establishment of an effective treatment both in MS and NAFLD.

\section{References}

1. Chalasani N, Younossi Z, Lavine JE, Diehl AM, Brunt EM, et al (2012) The diagnosis and management of non-alcoholic fatty liver disease: Practice 
Citation: Genel S, Aurelia C, Donca V, Emanuela F (2015) Is the Non-Alcoholic Fatty Liver Disease Part of Metabolic Syndrome? J Diabetes Metab 6: 526. doi:10.4172/2155-6156.1000526

Guideline by the American Association for the Study of Liver Diseases, American College of Gastroenterology, and the American Gastroenterological Association. Hepatology; 55: 2005-2023.

2. Prashanth M, Ganesh HK, Vima MV, John M, Bandgar T, et al. (2009) Prevalence of nonalcoholic fatty liver disease in patients with type 2 diabetes mellitus. J Assoc Physicians India 57: 205-210.

3. Williams CD, Stengel J, Asike MI, Torres DM, Shaw J, et al (2011) Prevalence of nonalcoholic fatty liver disease and nonalcoholic steatohepatitis among a largely middle-aged population utilizing ultrasound and liver biopsy: a prospective study. Gastroenterology 140: 124-131.

4. Kotronen A, Peltonen M, Hakkarainen A, Sevastianova K, Bergholm R, et al. (2009) Prediction of non-alcoholic fatty liver disease and liver fat using metabolic and genetic factors. Gastroenterology 137: 865-872.

5. Yki-Järvinen $H$ (2014) Non-alcoholic fatty liver disease as a cause and a consequence of metabolic syndrome. Lancet Diabetes Endocrinol 2: 901-910.

6. Dowman JK, Tomlinson JW, Newsome PN (2010) Pathogenesis of nonalcoholic fatty liver disease. QJM 103: 71-83.

7. Fabbrini E, Sullivan S, Klein S (2010) Obesity and nonalcoholic fatty live disease: biochemical, metabolic, and clinical implications. Hepatology 51: 679689

8. Cohen JC, Horton JD, Hobbs HH (2011) Human fatty liver disease: old questions and new insights. Science 332: 1519-1523.
9. Chen $\mathrm{SH}, \mathrm{He} F$, Zhou HL, Wu HR, Xia C, et al. (2011) Relationship between nonalcoholic fatty liver disease and metabolic syndrome. J Dig Dis 12: 125-130.

10. Anstee QM, Targher G, Day CP (2013) Progression of NAFLD to diabetes mellitus, cardiovascular disease or cirrhosis. Nat Rev Gastroenterol Hepato 10: 330-344.

11. Ratziu V, Bellentani S, Cortez-Pinto H, Day C, Marchesini G (2010) A position statement on NAFLD/NASH based on the EASL 2009 special conference. J Hepatol 53: 372-384.

12. Pacifico L, Nobili V, Anania C, Verdecchia P, Chiesa C (2011) Pediatric nonalcoholic fatty liver disease, metabolic syndrome and cardiovascular risk. World J Gastroenterol 17: 3082-3091

13. Ferreira VS, Pernambuco RB, Lopes EP, Morais CN, Rodrigues MC, et al (2010) Frequency and risk factors associated with non-alcoholic fatty live disease in patients with type 2 diabetes mellitus. Arq Bras Endocrinol Metabol 54: $362-368$

14. Nascimbeni F, Pais R, Bellentani S, Day CP, Ratziu V, et al. (2013) From NAFLD in clinical practice to answers from guidelines. J Hepatol 59: 859-871.

15. Hijona E, Hijona L, Arenas JI, Bujanda L (2010) Inflammatory mediators of hepatic steatosis. Mediators Inflamm 2010: 837419. 